\title{
Identifying treatment schemes for fruit and vegetable wash-water.
}

\author{
Richard G Zytner \\ University of Guelph, Canada
}

\begin{abstract}
The fresh-cut fruit and vegetable sector continues to expand as consumers are looking for fresh yet convenient ready-to-eat foods. The driving force behind this increase is the many health benefits that researchers continue to report for fruits and vegetables. Investigations have shown that the processing of fresh-cut fruits and vegetables generates about $5 \mathrm{~L}$ of wastewater (wash-water) per $\mathrm{kg}$ of produce, whether it is fruit, leafy greens or root vegetables. This wash-water requires treatment prior to disposal or recycling. Generic water treatment units are too expensive for most small to medium sized operations, which require more affordable technologies. Even though there are a variety of treatment technologies available, the challenge for producers and regulators is that the selection of the appropriate treatment technology is difficult, and tools are needed to help make the decisions. Data for the research was collected by collecting samples from two different types of operation, washing and washing and processing. Various bench scale treatment processes where then tested, including settling, coagulation and flocculation with settling, centrifuge, dissolved air flotation, electrocoagulation, screening and hydrocyclone. The decisions tools developed decision matrices to summarize the removal effectiveness of the different treatments for typical wastewater parameters and serve as a reference tool in understanding wash-water treatment technologies and their effectiveness in treating various wash-waters. The data was then further analyzed to develop predictive correlations for raw wash-waters, followed by the development of models that identified which treatment process worked best for the type of wash-water being handled. Combining the decision matrices, correlations, and models also show the potential for water reuse. For example, wash-water high in suspended solids can be treated by electrocoagulation and settling. The tools provide information that growers, government and consultants can use in determining treatment options that were not previously available or studied in literature.
\end{abstract}

Keywords: Fruits, Vegetables, Food poisoning, Health, Hygiene, Food safety

\section{Introduction}

Why Do You Need to Sanitize Fruits and Vegetables?

Fruits and vegetables go through a quite a journey before they make it into your crisper drawers, and along the way, they pick up things you don't want to be eating, including wax, dirt, and chemical pesticides. They can also harbor pathogens that cause food borne illness, an issue that affects one in six Americans every year according to the Centers for Disease Prevention and Control (CDC).

How to Safely Sanitize Fruits and Vegetables?

Effectively washing your fruits and veggies before eating them is actually a lot easier than you might assume. Here's how to do it.

DO wash your hands first. You don't want to accidentally transfer germs from your hands to your produce before and after sanitizing them, since that would be quite counterproductive. Follow the established guideline of washing your hands with soap and water for a full $20 \mathrm{sec}$, and then you can go grab your food items. Hand sanitizer is sufficient here too.

DON'T wash produce items before you're ready to eat them. Moisture is a perfect habitat for bacteria to prosper in. If you sanitize fruits and vegetables before you're ready to consume them, you run the risk of creating an environment where additional bacteria can thrive. This bacteria isn't necessarily dangerous, but it can speed up the process of your produce going bad, which is not ideal in the best of times and particularly so when you're trying to make as few trips to the grocery store as possible.

DO use just cold water. There has been a lot of information spreading around about proper sanitization methods at home in light of the coronavirus. If you've been paying attention, then you've probably seen recommendations that you sanitize fruits and vegetables in soap or special produce rinses, but a lot of experts-including the U.S. Department of Agriculture-are saying that's a bad idea.

Many fruits and vegetables are porous, meaning that it's easy for something you apply on the outside to seep its way to the inside. If consumed, soaps, even gentle ones, can cause stomach distress and are not approved for use on food by the FDA. As for produce sprays and rinses, there's no evidence that they're more effective than water in cleaning your produce and little research has been done in their clinical safety.

DON'T forget to be thorough. A cold water rinse is all that you should need to make your fruits and veggies safe to eat, but technique is important here. If you're 


\section{Short Communication}

sanitizing lettuce, cabbage, similar leafy greens, remove the outer layer of greens before sanitizing. Remove the leaves from other fruits and vegetables too before cleaning, and get rid of any damaged or bruised spots, which can harbor bacteria.

For items like apples, potatoes, carrots, and other hard fruits and vegetables, use a vegetable brush during the cold water rinse to scrub the surface. For softer produce items like berries, tomatoes, and mushrooms, use your hands to gently but firmly rub the item while it's being rinsed.

DO consider a baking soda bath if you want to take it a step further. If cold water just doesn't sound like enough to you, then an extra step would be a baking soda bath. A recent study published in the Journal of Agricultural and Food Chemistry found that a baking soda soak was the most effective method of removing pesticide residue from apples, even when compared to Clorox bleach (which, it's worth noting, should never be used on food).

To do a baking soda bath, clean and sanitize your kitchen sink, and then fill it with cold water. Next, add your baking soda-about a teaspoon should be sufficient, though you can add up to a few tablespoons if you prefer. Add your fruits and vegetables to the bath and let soak for $12 \mathrm{~min}$ to $15 \mathrm{~min}$, then scrub harder items and those with rinds with a vegetable brush. Thoroughly dry all items before eating.

Note that the above point about not washing your fruits and vegetables before you're ready to eat them still stands. Only use a full baking soda bath in the sink if you're planning on using a lot of produce items; otherwise a bowl of water with a pinch of baking soda will be enough for one or two items at a time.

Do Some Fruits and Vegetables Need To Be Sanitized More Than Others?

A lot of Americans are more worried right now about figuring out how to pay their rent or mortgage than they are about spending extra on organic produce. That being said, it's worth paying attention to which produce items are more likely to be contaminated by pesticides so that you know where to really focus your sanitization efforts.

Every year, the Environmental Working Group releases a list of the Dirty Dozen (produce items likely to be highest in pesticides) and the Clean Fifteen (produce items you don't have to worry as much about). When you sanitize fruits and vegetables, keep this distinction inmind.

The Dirty Dozen (in order): Strawberries, spinach, kale, nectarines, apples, grapes, peaches, cherries, pears, tomatoes, celery, potatoes, hot peppers.

Buy these produce items organic if you can, and always take extra care when rinsing them to remove unwanted substances.

The Clean Fifteen (in order): Avocados, sweet corn, pineapple, onions, papaya, sweet peas (frozen), eggplants, asparagus, cauliflower, cantaloupes, broccoli, mushrooms, cabbage, honeydew melon, kiwi.

\section{Conclusion}

There is a evident like germs can enter human body through various means in which one of the among was through raw material that are taken by the humans in raw form or by cooking. These are main cause for food poisoning and it's clear that clean and maintaining hygiene is the only way to protect ourselves from these hazards.

\section{Biography}

Richard G Zytner joined the School of Engineering, University of Guelph in 1991 after working in industry with Clayton Environmental Consultants. Since then he has taught both undergraduate and advanced graduate courses on water and wastewater treatment. His research interests have centered on soil remediation and wastewater treatment technologies. These projects have taken him several times to Germany on research leaves. Currently he is working on the fresh cut fruit and vegetable sector, with his research group looking at ways to treat both agri-food wash-water and wastewater, with the goal of reducing fresh water consumption. He has published and presented over 130 papers and is an Associate Editor with the Canadian Journal of Civil Engineering.

\section{References}

1. Mundi G, Zytner R G and Warriner K, et al. Fruit and vegetable wash-water characterization, treatment feasibility study and decision matrices. Canadian Journal of Civil Engineering 2017.

2. D Alharbi KK, Lau V and Liang C, et al. Treatment of spent wash water derived from shredded lettuce processing using a combination of electrocoagulation and germicidal ultraviolet light. Food Quality and Safety 2017.

3. Moore A, Zytner R G and Chang S, et al. Potential water reuse for high strength fruit and vegetable processor wastewater with a membrane bioreactor (MBR). Water Environment Research 2016;88(9):852870.

4. Lam K, Zytner R G and Chang S, et al. Treatment of high strength vegetable processing wastewater with a sequencing batch reactor. Journal on Agricultural Engineering 2015;2(1):30-38.

Mundi G S and Zytner R G. Effective solid removal technologies for wash-water treatment to allow water reuse in the fresh-cut fruit and vegetable industry. Journal of Agricultural Science and Technology 2015.

\section{*Correspondence to: \\ Richard G \\ University of Guelph \\ Canada \\ Email: rzytner@uoguelph.ca}

